International Journal of Biomedicine I June 2019 - Volume 9, Issue Suppl_1: Abstracts From the Second Russian International Conference "Cryo-electron microscopy 2019: achievements and prospects"

\author{
ORAL ABSTRACT PRESENTATIONS \\ SESSION TITLE: STRUCTURE AND FUNCTIONS OF THE TRANSCRIPTION AND TRANSLATION \\ APPARATUS OF THE CELL
}

DOI: 10.21103/IJBM.9.Suppl_1.OR2

\title{
Abstract OR-2: Investigating Interaction of Pioneering Transcription Factors with Nucleosomes Using Cryo-Electron Microscopy
}

\author{
Svetlana Dodonova, Patrick Cramer \\ Max Planck Institute for Biophysical Chemistry, Göttingen, Germany
}

Background: Specific regulation of gene expression determines cellular life and fate. Hundreds of transcription factor proteins (TFs) play critical role in this process. Typically, TFs bind short DNA motifs and then alter expression of their target genes. Majority of cellular DNA is associated with histones, as nucleosomes and higher order arrays, which occlude binding of most TFs. Only a minority of TFs (the pioneering TFs) are able to bind compact chromatin, and thus play a major role in altering gene expression and changing developmental programs. For instance, Oct4, Sox2 Klf4, c-Myc are highly expressed in embryonic stem cells and are able to drive differentiated somatic cells into pluripotent state. A recent study systematically explored interactions between the nucleosome and $220 \mathrm{TFs}$, and showed that most TFs preferably bind free DNA, and very few are able to bind nucleosomes in vitro. This study has also provided a selection of DNA-templates selected for ability to form nucleosomes and promote binding of a TF of interest.

Results and methods: Currently our mechanistic understanding of how pioneer TFs interact with chromatin is rather poor. Our structural knowledge in that area is limited to individual TFs or TFs bound to short DNA fragments. Thus, we aimed at structural characterization of pioneer TFs in the context of a nucleosome. We have formed a complex of a nucleosome on a DNA template from with a pioneer TF from purified individual components and have solved the structure by cryo-EM. We have confirmed the binding of a pioneer TF to the nucleosome by electrophoretic mobility shift assays.

Conclusions: We report the first structure of a nucleosome and a pioneer TF. Our structural and biochemical results illustrate a possible role of a pioneer TF in nucleosome destabilization and shed light on how such TFs can interact with chromatin.

Key Words: nucleosome, pioneer factors, cryo-EM

International Journal of Biomedicine. 2019;9 Suppl 1: S6. doi: 10.21103/JJBM.9.Suppl_1.OR2

(C)2019 International Medical Research and Development Corporation 